\title{
Evaluation of Syndromic Surveillance in Detecting Hepatitis A in Los Angeles County
}

\author{
Michael Lim*, Emily Kajita and Bessie Hwang
}

Public Health, Los Angeles County, Los Angeles, CA, USA

\section{Objective}

To create a hepatitis A virus (HAV) syndrome category with which to monitor emergency department (ED) visits for situational awareness during a currently emerging Hepatitis A community outbreak in Los Angeles County (LAC), and to evaluate its usefulness.

\section{Introduction}

In early 2017, HAV outbreaks were identified in San Diego County (490 cases) $)^{1}$ and Santa Cruz County $(73 \text { cases })^{2}$ in California, affecting primarily the homeless and/or illicit drug users. As of October 10, 2017, LAC had identified 12 outbreak-related HAV cases. Due to LAC's proximity to San Diego County, and its own large homeless population, the syndromic surveillance team of the LAC Department of Public Health created a syndrome category and began querying its ED data to monitor for any increase in HAV-related visits.

\section{Methods}

ED data from 1/1/2017 to 10/10/2017 (CDC weeks 1-41) from all participating syndromic EDs in LAC were queried for patients who reported symptoms and signs of HAV. For comparison, ED data from calendar year 2016 was also queried. The query consisted of key word searches primarily within the chief complaint field, and, if available, the diagnosis and triage note fields. Based on the Centers for Disease Control and Prevention (CDC) clinical description of hepatitis $\mathrm{A}^{3}$, the HAV syndrome category was defined as: jaundice (or elevated liver function tests) AND nausea or vomiting. Any ED visit that mentioned a diagnosis of hepatitis A also met the syndrome criteria. The resulting line lists were reviewed, and the query was periodically refined to exclude visits unrelated to hepatitis A (e.g., previous history of or vaccination for hepatitis A, other forms of hepatitis, and neonatal jaundice). The syndromic system was also queried for records that matched the 12 LAC cases by hospital and admission date. In addition, the chief complaint, diagnosis, and triage note fields were reviewed for any mention of homelessness or intravenous drug use.

\section{Results}

The syndromic system detected $158 \mathrm{ED}$ patients meeting the HAV syndrome category criteria. Of these, $12.7 \%$ had a diagnosis of HAV, $53.8 \%$ had jaundice, $36.7 \%$ had elevated liver enzymes, $65.2 \%$ had nausea, and $65.8 \%$ had vomiting. In 2016, 170 ED patients who met the syndrome criteria were detected: $8.2 \%$ had a diagnosis of HAV, $64.1 \%$ had jaundice, $32.4 \%$ had elevated liver enzymes, $63.5 \%$ had nausea, and $71.2 \%$ had vomiting. In both years, no indications of homelessness or IDU were found.

Of the 12 LAC confirmed HAV outbreak-related cases, three did not go to a hospital, and thus did not have any syndromic data. Two went to non-participating syndromic EDs, but medical chart review showed that they would not have met the syndrome criteria. Of the remaining seven, all went to a participating syndromic ED, and three met the syndrome criteria, but none had any mention of homelessness or IDU.

\section{Conclusions}

No major change was seen in the trend of HAV syndrome visits in 2017 compared to 2016 (Figure 1). One of the challenges in monitoring HAV incidence is that the clinical signs and symptoms are very general, and may be shared with many other conditions. An emerging outbreak may not be detected above background levels unless the increase in ED patients with HAV is large, or consolidated over time. Variability in data quality in the free text fields such as chief complaint and triage notes may be problematic. Cases will be missed if data fields are not fully and accurately documented, and also if patients didn't go to a participating syndromic hospital, or to a hospital at all. Though many syndromic hospitals now report diagnosis information, such information may be delayed, waiting for lab results. Using a stricter syndrome definition, such as requiring a specific diagnosis of HAV, may result in underreporting, but may provide a more accurate baseline for detecting increases and monitoring trends.

While the query relied primarily on ED chief complaint, diagnosis and triage notes also proved useful in detecting HAV syndrome visits. None of the confirmed HAV cases that were known to be homeless had any mention of homelessness. The lack of any records indicating homelessness or IDU may indicate that these conditions are not currently reliably captured in the syndromic extract of ED patient records.

LAC will continue to monitor for increases in HAV syndrome ED visits as a surrogate measure of HAV spread in the community. Syndromic surveillance, despite its limitations, remains a valuable complement to electronic lab reporting and other traditional reporting mechanisms.

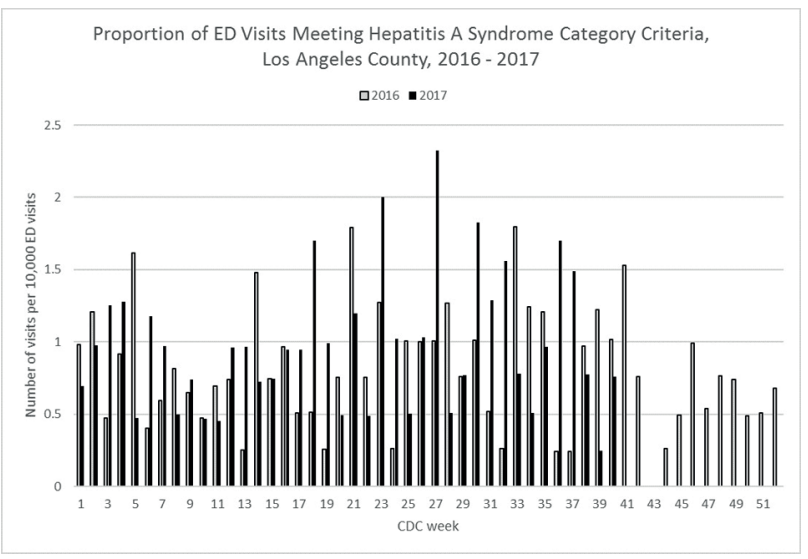

Figure 1. Proportion trend graph of HAV syndrome ED visits.

\section{Keywords}

syndromic surveillance; hepatitis A; homeless 


\section{ISDS 2018 Conference Abstracts}

\section{References}

1. San Diego Hepatitis A Outbreak, 2017 [Internet]. San Diego, CA: San Diego County Health \& Human Services Agency [cited 2017 Sep 21]. Available from: http://www.sandiegocounty.gov/content/sde/hhsa/ programs/phs/community_epidemiology/dc/Hepatitis_A.html

2. Santa Cruz Hepatitis A, 2017 [Internet]. Santa Cruz, CA: County of Santa Cruz Health Services Agency [cited 2017 Sep 21]. Available from: http://www.santacruzhealth.org/HSAHome/HSADivisions/ PublicHealth/CommunicableDiseaseControl/HepatitisA.aspx

3. Hepatitis A, Acute 2012 Case Definition [Internet]. Atlanta, GA: Centers for Disease Control and Prevention National Notifiable Diseases Surveillance System [cited 2017 Sep 21]. Available from:https://wwwn.cdc.gov/nndss/conditions/hepatitis-a-acute/casedefinition/2012/

*Michael Lim

E-mail: milim@ph.lacounty.gov 